\title{
NINTH DISCUSSION SESSION
}

(Monday afternoon; 11 September, 1972)

(Following Sections 4 and 5, of Plavec's review paper)

\section{Chairman: D. M. POPPER}

Hall: If $\mathrm{AX}$ Mon is losing mass at such a very high rate, even though it is relatively difficult to determine an orbital period accurately from spectroscopic observations, you might be able to put an upper limit on the amount of mass exchange by looking for a change in the period. Is there any known variation in the spectroscopically determined period?

Plavec: No, we thought of that. You get a fairly large change of period that should be detectable over fifty years; but for spectroscopic binaries period determination is much less accurate than for eclipsing binaries. When AX Mon was rediscussed by Anne Cowley, the change introduced by the improved observations was larger than the real change in the orbital period.

Cowley: On the other hand, the original orbit for AX Mon had a period of $235^{\mathrm{d}}$ and I looked at those observations rather carefully, to see if they could be fitted to the revised orbital elements. I decided that they could be.

Smak: One thing bothers me, namely, is it possible - when we are talking about rapid mass transfer - to have the mass-collecting component look like a normal star, say a B-type star? Let me quote a crude estimate concerning the amount of matter to fall on the surface of such a star. Assuming that the rate is $5 \times 10^{-5} M_{\odot} \mathrm{y}^{-1}$, and that the star has a radius of $4 R_{\odot}$, that only $1 \%$ of the mass transferred falls on the star, and that this matter is distributed uniformly over its surface, we get a flux of about $10^{20}$ incoming particles $\mathrm{s}^{-1} \mathrm{~cm}^{-2}$ of the stellar surface. This seems quite a lot, even without mentioning the problem of energy. I haven't made any estimates on that, but I believe that many of us here feel quite strongly that in the case of such major infall of material, its radiation must obliterate completely the spectrum of the central star.

Concerning T CrB, however, I feel that Dr. Plavec's interpretation could be the correct one. It may be noted that the theoretically predicted rate of mass transfer could probably agree (within the uncertainties involved) with the amounts of mass ejected by the star during its repeating nova-outbursts. I also have two minor comments to make on this system. First, according to a rediscussion by Paczyński (1965a) the limit on masses are $1.9 M_{\odot}$ and $2.6 M_{\odot}$. Second, if I remember, Kraft's estimates of the parameters of the 'blue' star were - at least partly - based on the assumption that the emission lines from the disk are due to the ionization of the disk by the central star, which may not be true.

Plavec: I know about Paczyński's paper and his values are included in my manuscript; they are not significantly different because the most important thing which has 
been established in both cases is that the blue component is most likely not a degenerate star. As to the behaviour of the mass-receiving star, I am equally puzzled as you, Smak. One thing is just to study the mass-losing star and come to this conclusion from which somehow I would like to escape, but I don't exactly know how. This is why I presented the case of terrible mass loss from the convective atmosphere. The other thing is whether this is a possible explanation for AX Mon. There certainly is something happening to the mass-accreting star, but not so terribly much. If this discussion stimulates some further investigation into the problem of the reaction of the massreceiving star, then it will have accomplished something.

Popper: Dr. Bath, you may give your summary.

Bath: I intend to discuss some work I've been involved with for the past four years relating to this problem of dynamical instabilities in semi-detached binary systems. The approach I have been using is somewhat different from that used by Dr. Plavec, and others, who have been using programs which, designed specifically to investigate longer time-scale thermal instabilities, employ the approximation that acceleration terms are negligible. Because of this difference of approach it may be that we are talking about somewhat different phenomena and using the same term, dynamical instability, to describe it.

The point of departure of my own approach has been exactly that point which Dr. Smak mentioned before lunch. That is, that dynamical instabilities in semidetached systems will, if they occur, be totally analogous to those in single stars and that one must therefore use the same methods as are employed in pulsation studies to analyse them. All the physical terms that arise in the single star problem will be relevant to the case of dynamical mass transfer in semi-detached systems. The only distinction between the two cases is the boundary condition. A single star is free to expand and contract at will, whereas the contact component in a semi-detached system is constrained by the potential fields to remain within some fixed volume defined by the Roche lobe. To simulate this constraint one must use a boundary condition which acts at the Roche lobe surface, and not on some fixed mass layer at the surface of the star. The major problem is to define exactly what this new boundary condition is and to apply it to stability tests of detailed model envelopes.

This brings in the problem of treating an essentially asymmetric system in which the constraining boundary acts in a simple way at only one point, the inner Lagrangian point, by employing spherically symmetric stellar models with an associated spherically symmetric boundary condition. This is a particular difficulty in the case of dynamical instabilities, in which hydrodynamic terms must be included.

In the case of the thermal instabilities described in Dr. Plavec's review, no hydrodynamic boundary condition need be employed. The physical boundary of the star is assumed to be the photospheric surface and mass is removed at such a rate as to keep the photosphere in contact with a spherical surface whose volume is equal to the Roche lobe at all times. We have seen that in some cases, in particular in stars with deep convective envelopes, it is impossible to remove mass at such a rate as to fulfil this condition. It appears that the faster mass is removed, the faster the photosphere 
expands. In the model described by Dr. Plavec the star goes through a phase when the photosphere overflows the lobe by something like $10 \%-20 \%$. Any attempt to remove mass at an even faster rate leads to numerical instabilities.

If one includes all the hydrodynamic terms, such an approach cannot be employed. For then an additional boundary condition must be introduced to control the acceleration equation at the Roche surface. This boundary condition affects the behaviour of the star only when overflowing the lobe. Rather than arbitrarily controlling the mass loss by the behaviour of the photosphere, which, after all, reflects only the position of a region where the optical depth has some specified value, which is really irrelevant in the hydrodynamic situation, one must control the mass loss by a boundary condition that reflects the flow properties of the real system.

In an attempt to simulate this in spherical models I have employed the boundary condition,

$$
\frac{\partial P}{\partial t}=0
$$

That is, the pressure is forced to be constant at all times, at some fixed radius, equivalent to the 'effective' radius of the Roche lobe. The justification for this is based on the argument that the equipotential surfaces, including the limiting surface of the Roche lobe, must be constant pressure surfaces in the static situation. Once an instability insues material will stream towards the second component, to be accreted, probably, as a disc. So long as the companion is well detached, and the amount of mass transfered is small, no increase in back pressure across the Lagrangian point will occur. Thus the outer critical equipotential will remain at constant pressure. This sort of boundary condition receives some support from more detailed treatments of the flow based on the similarity of the potentials in the vicinity of the Lagrangian point to a de Laval nozzle.

With this condition of constant pressure at a fixed surface one can use all the techniques and methods of stability theory in single stars but, since the boundary condition is Eulerian, the equations must be transformed to an Eulerian coordinate system. Some work based on this point of view has been completed using both linear and non-linear, and both adiabatic and non-adiabatic methods.

The linear adiabatic solutions indicate that, if the photosphere is initially in contact with the Roche lobe, then dynamical instabilities will occur in all stars with convective envelopes, that is, with any extent of ionisation zones in the surface. The growth times of the instabilities suggest an extremely rapid phenomena. A non-linear, but adiabatic, time dependent code has confirmed this, and shows that large temperature changes, and associated luminosity variations can be expected. These arise as a consequence of an increase in the temperature gradient in the superadiabatic region of the evnelope. For, as the outward flow builds up, material in the hydrogen and helium I zones recombines. It is just this recombination energy which heats the gas and drives the instability. Adiabatic cooling is not sufficient to keep the temperature gradients down to their initial values within the original static model. The regime where the adiabatic 
approximation must break down only occurs after considerable luminosity increase has occurred at the surface.

All these conclusions have recently been confirmed by a fully non-linear, nonadiabatic program, similar to that used by Christy in pulsation studies. Only the early stages have so far been followed, but a rapid rise in surface luminosity again occurs. This is true with both the boundary condition I have described, and a somewhat different, less constrained, condition.

How these instabilities relate to the observational problems we have been discussing remains to some extent obscure. That they may relate to the $U$ Gem phenomena, as originally suggested by Paczyński (1965b), is still possible from a theoretical point of view. Certainly the energy requirement is available in the alternate removal and reestablishment of the hydrogen and helium ionisation zones. Whether novae are in any way related to dynamical mass transfer, as Dr. Plavec mentioned, is still open to question also. Furthermore it is not clear how the instabilities I have been describing, driven by ionisation zones in the outer envelope, relate to the more 'structure' dependent instabilities that Dr. Plavec and others have found. A more rigorous analysis of the relevant boundary conditions that theoreticians are using to simulate an asymmetric situation with spherical models is required on the one hand, and a concerted effort by observers to find out exactly which components are changing in variable and explosive semi-detached binaries on the other.

$R$. E. Wilson: All of these results are consequences of your boundary condition, so that should be examined very closely. In a formal sense it's not surprising that the pressure gradient is zero at the surface, since the pressure itself is zero there.

Bath: No, not if you've got a reasonable initial model; not if you've got a detailed treatment of the photosphere. In the spherically symmetric case $P=g / k$ at the surface. The pressure is very small, but not zero.

Whelan: I think you've got to emphasize that the pressure is not zero at the surface of the star. The density is about $10^{-8} \mathrm{gm} \mathrm{cm}^{-3}$.

Bath: The pressure cannot be zero in the spherical case. As long as the pressure there is constant at a fixed radius, even if it is zero, this boundary condition is very, very important! And the difference between it and the free boundary condition is crucial.

R. E. Wilson: Yes, but aren't you effectively imposing a formal condition and then extrapolating it from regions of very low-energy processes down into regions of very high-energy processes and thus dictating the behaviour of much of the outer part of the star?

Bath: No, because all I'm doing in the non-linear models is putting an initial perturbation in the outer three or four zones where there are no energy sources at all. These outer zones communicate their motion, in accordance with the hydrodynamical equations, to regions which then drive the instability. They are driven by recombination in exactly the same way as in the model of planetary nebulae proposed by Paczyński and Ziołkowski (1968), and independently by Lucy (1967). Their model is just the same sort of dynamical instability but of single stars. Dynamical instabilities occur 
in single stars of very high luminosity, just because the energy from the hydrogen and helium ionization zones can, as they penetrate inwards, be converted into kinetic motion of the envelope.

R. E. Wilson: Can you demonstrate some direct way that your boundary condition is going to put important constraints, in a physical sense, on the value of the pressure gradient at some level deeper in the star?

Bath: Just by doing a linear analysis, that's all. Then you're tying a boundary condition into the solution of a linear differential equation, which thus determines the behaviour of the solution at the boundary.

Popper: I'm afraid this exchange is likely to get out of hand. I don't want to minimize its importance but I think clearly there are some difficulties which people working on this program will have to clear up.

Whelan: The important thing about using rotating polytropes is that you leave out some physics. It is more important to include the physics and leave out the rotation terms. I think the importance of Dr. Bath's work is that it shows that you cannot have these rapid mass transfers without worrying about the dynamical terms.

Popper: I think perhaps we should move on. Obviously this is a very pertinent field and maybe there will be some occasions to come back and have some more discussions on it. I think we'll get Dr. Plavec to go on with the next block of his subject.

Plavec: Let me make one final comment on that last discussion. It's no doubt that it's much better if analysis is done so that dynamical terms are taken into account and then you can discuss what happens on time scale much shorter than you can do if you use the normal evolutionary code.

(Following Sections 7 and 8 of Plavec' review paper)

Devinney: The light curve of $v \mathrm{Sgr}$ is quite peculiar for a long-period $\operatorname{star}\left(P=138^{\mathrm{d}}\right)$ because the light curve is characterized by a general light variation throughout the cycle. If my memory is serving me right, the light and radial-velocity curves are inconsistent in that the deepest minimum occurs when the radial velocity is increasing.

Plavec: Gaposchkin's (1945) photographic observations are still the only ones available, except for some reported in a short paper by Eggen et al. (1951) that essentially confirms Gaposchkin's picture. There is nothing else. The depths of minima are 0.15 and $0 \mathrm{~m} .08$, but the probable error of a single determination is $\pm 0 \mathrm{~m} .20$. It's true that the deeper minimum is the eclipse of the star we don't see. This other star is $3 M_{\odot}$ or so, sits on the main-sequence, and it is actually the star which has the larger surface brightness. So, if the eclipse is grazing, the helium giant of about $15 R_{\odot}$ or more eclipses, at the time of primary minimum, a small star which is probably of a higher effective temperature - namely. a B-type star. So there is no inconsistency, but of course the light curve should be reobserved.

Thackeray: If one examines Boyarchuk's list of 19 symbiotic stars, for the mean galactic latitude we find $|b|=17^{\circ}$, while for 13 of these objects with known radial velocities, 9 have mean radial velocities $|V|>50 \mathrm{~km} \mathrm{~s}^{-1}$. This is to be contrasted with the low latitude of the supergiant VV Cep stars. Thus we should regard the symbiotic 
stars as an older population than the VV Cep stars, which is perhaps relevant to Dr. Plavec's discussion.

R. E. Wilson: I have proposed a model for Cyg X-2 which involved a white dwarf accreting material from the G-type primary in this system (Wilson, 1970). The spectrum of Cyg X-2 has broad absorption lines, which are very interesting, because absorption lines give information about physical conditions in the vicinity of the stellar component near which they are produced, whereas emission lines may come from gas which is far from both components. These absorption lines are very broad, and indicate mass motions of the order of $1000 \mathrm{~km} \mathrm{~s}^{-1}$. One can eliminate broadening mechanisms other than mass motions. Since there must be a continuum against which we see the absorption lines, and the G-star is unlikely to be responsible, my model postulated an optically thick region of accreting material in the form of a disk or cloud around a white dwarf. It is interesting that Dr. Plavec mentioned a helium star as an accretion source because a helium star could be bright enough to serve as a continuum source for the absorption lines, yet faint enough to escape spectroscopic detection through its own lines. Therefore, a variation of the above-mentioned model involving a helium star might also be interesting to consider. It is very important to continue observing Cyg X-2, since Kraft's interesting observations were discontinued after only five or six night's work. Furthermore, the optical identification, which was only at the fifty percent confidence level several years ago, is now virtually certain.

Smak: Regarding various models for binary X-ray sources, it seems to me that in some cases it might easily be that the two parameters, namely the rate of infall of matter and the temperature, could be independent. If so, the shape of the X-ray spectrum could tell us about the mechanism involved, while the intensity could tell us about the rate of mass transfer. In the case of the Prendergast-Burbidge model, or - more generally - whenever circumstellar matter is involved, the two parameters are closely connected because of the essential role being played by the disk.

Regarding the absorption lines, mentioned by Dr. Wilson, I wish to recall that broad, shallow absorption lines of hydrogen are often observed in the spectra of $U$ Gem stars at their outbursts. To explain such cases, I feel that a continuous emission from optically thick circumstellar material may produce the background against which the absorption lines produced in the outermost parts are seen.

Bolton: I would like to make a few general remarks on X-ray sources. For almost all of the sources the X-ray spectra are not well enough known to allow us to determine the mechanism that produces the X-rays. This is because of several things including poor observations, lack of observations, and source variability. It is now known, however, that the binary sources, of which eight or nine are known, have generally flatter spectra than other sources. Attempts to fit the spectra of a variety of $\mathrm{X}$-ray sources with simple source models, such as synchrotron radiation, optically thin thermal bremsstrahlung, or thermal black-body radiation, have generally not been very satisfactory. Usually at least two models fit the spectra equally well, at least in the lower-energy region from $2 \mathrm{keV}$ to $20 \mathrm{keV}$. For only a few X-ray sources is there enough high-energy data so that anything can be said about their spectra above 
$20 \mathrm{keV}$. Let me repeat that the spectral situation is very complicated. In Cyg X-1 it may be that there are two or three different source regions with different kinds of spectra and variability. Sorting this all out will require much better X-ray data than are currently abailable. I would like to make some remarks on Cyg X-1 in particular. I think in light of Dr. Hutchings earlier remarks on the antiphase motion of He II $\lambda 4686$ that my interpretation of that motion is very much open to question. This would not, however, change the basic interpretation of the system. The mass function of the B0 Ib star requires that $M_{2}>4.8 M_{\odot}$ if $M_{\mathrm{BOIb}}=20 M_{\odot}$. The observational requirement that there be no eclipse of the B0 Ib star raises the lower limit to $M_{2}>5.4 M_{\odot}$ for $M_{1}=20 M_{\odot}, \log g=3.3$, and a distance of $2 \mathrm{kpc}$. Both of these lower limits are comfortably above the upper limits on the masses of neutron stars and white dwarfs.

Finally, I would like to point out that the derived eccentricity of 0.09 is quite large for a binary system if this period and primary star of this spectral type. I have checked carefully to see of the eccentricity could be spurious, but I now believe that it is not. This eccentricity is consistent with the behaviour of the emission lines, and it is possibly telling us something about the evolutionary state of the system.

Bopp: If I might make a few comments on Cyg X-2, as I recall from a paper by Kraft and DeMoulin (1967), the radial velocities did not yield a consistent period. The object certainly did not seem to be a double-lined spectroscopic binary, as several authors had hoped, with the HeII emission being produced by one component and the hydrogen absorption lines by the other. The final comments of Kraft and De Moulin concerning binary motion showed them to be dubious regarding it.

We have obtained some observations of Cyg X-2 in the past year, and the behaviour of the system seems to be quite different from what it was at its discovery in 1967. Photometrically there are no variations, in contrast to the results of Kristian et al. (1967). Recent spectra obtained at McDonald show that C III - N III emission at $\lambda \lambda 4640-50$ has suddenly appeared. The HeII line has developed a P Cyg profile, and the radial velocity of the $\mathrm{He}$ II emission seems to be constant.

Popper: What is the optical apparent magnitude of Cyg X-2?

Bopp: About 14.5 - and the optical identification is quite certain.

Bath: Do you suppose that the orbital eccentricity arises from the mass loss? Is it continuous mass loss?

Bolton: I think the eccentricity is a result of mass loss, but this would have to be on a time scale comparable to or less than the period of the binary system.

R. E. Wilson: I would like to mention that there is another binary for which a black hole might be indicated. I have done some work on BM Ori and the model in this case is very similar to that of $\varepsilon$ Aur. It involves the transit of an inclined disk with a transparent central opening. The arguments are not identical in the two cases but again I come to the conclusion that there is likely to be a black hole in this system. Now in the case of $\varepsilon$ Aur it is difficult to make a definitive comparison between the predicted and the observed optical light curves because the primary is intrinsically variable and there are fluctuations of the order of $0^{\mathrm{m}} .2$ or almost that large, in the F0 supergiant primary. The light curve of BM Ori (Hall and Garrison, 1969) shows a similar scatter, 
but in this case it arises from the difficulty of observation. It is very difficult to do photometry of this system because nebular emission from the trapezium affects measures made with broad-band filters very severely. It is important now to obtain a very precise light curve for the primary eclipse in which one goes to the greatest extremes possible to eliminate the background light of the nebula.

Plavec: The case of BM Ori of course is the most difficult problem. It is an eclipsing binary and is the faintest star in the Trapezium. The period is $6 \mathrm{~d}$, so you have two serious objections to your picture. First the period is a bit terribly short for producing a black hole; second, the Trapezium is a classic example of a very young, young as possible, multiple system. Now you say that this very latest stage of evolution is present there. Again, it's possible, but I would say that probably it would be wise to try to find other explanations. This really is a good model but these two objections from the point of view of stellar evolution, I consider serious.

R. E. Wilson: Well, I've listed these two objections, which are also objections of my own, in the paper, but of course we have similar problems with Cyg X-1 and I personally find it very difficult not to believe the case for Cyg X-1. By implication, therefore, this kind of objection is to be treated very carefully at this stage.

Smak: If I understand properly, there are at least two crucial points involved. First, we are looking for a binary with sufficiently well determined masses or limits on masses which are large enough to exclude the possibility of the invisible object being a white dwarf or another low-luminosity star. Secondly, however, we are looking for some details in the light curve which definitely cannot be explained by all other effects we have been talking about during the last few days, but could be explained by some effects due to the black hole. I am sorry to be pessimistic and sceptical at this point, but it seems to me that if a really convincing evidence for a black hole is ever to be presented at a symposium on binaries, the title of this symposium will more likely be: 'Binaries Without Circumstellar Matter'!

R. E. Wilson: The light curve of $\varepsilon$ Aur has a flat bottom, which is usually taken as the fingerprint of a total eclipse. There cannot be a total eclipse in this system, and I have tried to show that the light curve can only be produced by a thin disk, highly inclined (inclination $89^{\circ}$ ) with a central opening through which the F0 supergiant can shine (Wilson, 1971). This model will reproduce the light curve and the spectroscopic features. No other model but Dr. Huang's will do so - and that meets certain difficulties. The radial velocities of certain satellite lines are in very good agreement with the model (Kuiper et al. 1937). The secondary mass, which holds the ring together is not veiled, as in earlier models, but should be in full view. Since it has not yet been detected at any wavelength, its luminosity must be far less than normal for its mass. Finally, since that mass is of the order of $12 M_{\odot}$, it cannot be a white dwarf or a neutron star, and seems, therfore, to be a black hole.

Herczeg: Do you think that your model has definitively shown the existence of a black hole in $\varepsilon$ Aur?

$R$. E. Wilson: In effect, I say so in my paper.

Huang: Dr. Wilson mentioned my model of $\varepsilon$ Aur. My philosophy is to allow a 
great amount of freedom of thought in the interpretation of observations. Everyone can propose a model from his own perspective. We should produce as many models as we possibly can, and then, after twenty years, we can combine the best parts of these models together, and form a better one. I think every model of $\varepsilon$ Aur we have now has some merits - and some defects - we should let time take care of them. After all, stellar events took millions of years - we can afford to be patient.

Bolton: It is my opinion that given what we know of the physics it will never be possible to point to a specific system and say that that system contains a black hole. As I understand the theory a black hole has three characteristics: (1) a certain mass, (2) a radius defined by the mass (3) no luminosity. The only way that a black hole will call itself to your attention is through material falling into it, and that is only likely to be important in a binary system. Mass accretion by a black hole in a binary system may produce $\mathrm{X}$-rays, but the accretion process will probably produce a disk around the black hole. This disk will yield a spurious radius in any eclipse measurement, and if seen edge on, may block the X-ray emission. Thus about all we can hope to do is to detect a number of massive underluminous objects and hope that this collection of objects will show some regularities that can be theoretically explained.

Underhill: What kind of mass do you require in a black hole?

Bolton: Well, if you can believe the theories of such people as Cameron and Ostriker, then neither a neutron star nor a rotating white dwarf can be more massive than about $3 M_{\odot}$. Under very special and unlikely conditions a rotating white dwarf could be a bit more massive than this.

R. E. Wilson: I would like to point out that our faith in the law of conservation of energy is based on the fact that we have never seen it violated. It is not based on any original principle that someone found engraved on a stone. The hypothesis was made that energy is conserved; people have made experiments and failed to find that it is not conserved. Therefore, we believe energy is conserved. I say all this to emphasize that ideas of this kind are accepted eventually because of the weight of overwhelming auxiliary evidence, which accumulates over many decades and centuries.

Biermann: You might be able to determine the existence of a black hole from the high-energy X-ray spectrum.

Batten: I sometimes find myself wondering if we shouldn't draw a lesson from quite recent history. Neutron stars were talked about for about thirty years before pulsars were discovered, and when pulsars were discovered they were such a surprise, we didn't believe at first that they were neutron stars. If black holes ever are discovered, might they not be discovered by some completely unpredicted property? I think that if and when they are discovered, it will be beyond reasonable doubt what they are, and as long as there is reasonable doubt, I prefer to adopt the principle: entities should not be multiplied unnecessarily.

Popper: Well, since you are in a philosophical vein, let me put it another way. Nature in general is more imaginative than human beings!

Devinney: The question was raised: will we ever find binaries that are well behaved in which we may find some evidence for black holes? There are systems like $\delta$ Gem, 
which has a large mass function although the visible component is an apparently normal A-type star. Perhaps amongst systems of this kind, a black hole may be found.

Popper: Any further comments on this topic of crazy stellar objects? If not then I think we should open up the floor for general comments on Dr. Plavec's presentation.

Oliver: I have a question for Dr. Plavec that does not involve black holes or X-ray sources. There exists a group of binary systems that have in the past been called Algollike systems with undersized subgiants. We've talked about them already in this Symposium, and we hope to give more details tomorrow.* I would like to hear your comments now on their evolutionary status. The mass ratios in these systems seem to be near unity. The secondary star, usually like a K0 subgiant, may be either more or less massive than the hotter star. The secondary does not fill its Roche lobe, and there are rather too many of them with mass ratio close to one. Can you say anything about the mass transfer that seems to be going on in these systems?

Plavec: No, I can't. I, too, am puzzled. Many years ago I studied period changes in eclipsing binaries and I found that if one component of the system is off the mainsequence, there are period changes. If so, and if we accept that period changes always indicate mass transfer, then we must conclude that all giants are losing mass - which would be horrible to accept in general.

Popper: Now we can proceed with some of the listed topics. Dr. Walter, would you like to present your remarks on the period-eccentricity relation?

Walter: Among the mutual relations between the components of binaries from which we may get important information one should not forget the relation between periods of revolution and eccentricities of the orbits. It is a widespread opinion that this relation is only a statistical one in the sense that in general for systems with close components the orbits are circular and, for larger periods of the systems, the orbits pass over to elliptic ones, and that only the mean values of the eccentricities, for a large number of systems, are larger, the longer the periods.

But this is a rather superficial view, and I must criticize people who worked on the problem in recent years or reported on it for not having studied older literature. They are not aware of the fact that, with large probability, for main-sequence stars of spectral types B9 and later and within distinct regions of periods, the relation between periods and eccentricities is very close. The observations show that, depending on the physical propertics of the components of the systems, the period-eccentricity relation has a quite characteristic appearance.

The details of the relations between period and eccentricity for systems containing stars within certain spectral-type ranges are shown in the illustrations of my three papers (Walter, 1950, 1951, 1954). Recently I repeated some of this work, using Batten's (1967) catalogue. The number of systems available for these investigations increased only modestly in the two decades since the older Lick catalogue (Moore and Neubauer, 1948), but the relations suggested in my earlier work no longer seem valid for systems containing main-sequence stars of spectral types B8 and earlier.

* See p. 279. 
Spectroscopic binaries containing primary components of spectral type F8V to K5V and showing only one spectrum have orbits that are circular, or nearly so, if $P<10 \mathrm{~d}$. If $10 \mathrm{~d}<P<25 \mathrm{~d}$, there is a close correlation between period and eccentricity, which breaks down for $P>25 \mathrm{~d}$.

Binaries containing main-sequence components in the range of spectral types B9-A7 show a more complicated relationship. Those containing stars of nearly equal masses can be divided into two groups, $a$ and $b$, which differ in the period at which circular orbits give way to elliptical ones. Systems with very small mass functions seem to have the same maximum value of the eccentricity as do those containing equal stars. Moreover, features in the $(P, e)$ relation for these stars appear to be related to the rotational velocities of the A-type components. In some systems, the A-type component cannot be forced into synchronous rotation. Systems with intermediate mass functions also show a division into two groups. It is of interest that $\alpha \mathrm{CrB}$, which belongs to group $a$ in this class does indeed rotate much faster than synchronism would require. It is located in the $(P, e)$ diagram by the component of smaller mass.

Here I would like to touch only shortly on the theoretical aspects. My earlier statement that, because of the effects of tidal oscillations of the components, elliptic orbits should have a tendency to be transformed to circular ones, was independently put forward and improved by Zahn (1966) by means of detailed model computations. But, disregarding the existence of the above mentioned $(P, e)$ relation, this author did not realize the fact that as well an opposite tendency, one by which the eccentricities should grow with time, must be assumed in order that, as a result of an equilibrium between the two opposite influences, orbits may exist with the observed eccentricities. In one of my cited papers I have shown that differential rotation of binary components like that known for the Sun could increase orbital eccentricity. In the meantime it has come to seem very probable that magnetic fields exist in Algol systems connecting the components as I mentioned earlier in this Symposium.* Therefore one could imagine that besides gravitational forces, magnetic fields are also important for the transfer of rotational energy from the components into the orbit and that the bifurcation in the $(P, e)$ relations found for A-type stars might be caused by different combinations of the signs of the magnetic fields on the two components.

In any case one should think that observations of the orbits of spectroscopic binaries may give us information which are important for the study of the mutual connections between the components and the properties of main-sequence stars.

If one looks at the diagrams in my papers, one may still have some doubts of the statistical significance of the $(P, e)$ relation. I believe it would be very important to enlarge the observational material of spectroscopic orbits of main-sequence systems not only in order to ensure the reality of that relation but also to make an observational basis for detailed studies. It is a task which may be done by spectroscopists without outstanding instrumental means, since there are many stars of apparent 
magnitude between $7^{\mathrm{m}}$ and $8^{\mathrm{m}}$, among them over thousand A-type stars which have not yet been investigated for their binary characteristics. According to the probability of frequency of spectroscopic binaries, there should be many systems of binaries among them which could contribute to a comprehensive filling up of the $(P, e)$ diagrams. It is conceivable that, without an actual object and physical purpose in mind, spectroscopists during the last years and decades had little interest in further enlarging the number of spectroscopic systems. I would like to encourage them to continue work which I believe will be urgently needed in future by theoreticians.

Bolton: While I would number myself among the doubters as far as the reality of the effects you have described, I would like to echo and amplify upon your comments about the need for getting more statistical data. For the past two years, I've been using the 74-inch telescope of the David Dunlap Observatory during bad weather to look at bright early-type stars. I have found or confirmed literally dozens of B-type and A-type spectroscopic binaries, between second and fifth magnitude, which have no orbits.

Hill: We are doing the same thing at the Dominion Astrophysical Observatory, and can confirm Boiton's statement.

Wright: At noon, I received a letter from Dr. Aller. He was to have been present here but was prevented at the last minute. He would like some of his views to be presented to you. He particularly wants to stress the limitations imposed by kinetic theory on the behaviour of the gas in rings, shells, jets, etc. around binary systems. He cannot follow at all the justification of particle dynamics which, it seems to him, must fail by many orders of magnitude. The circumstellar matter should have high thermal and electrical conductivity, and he emphasizes the possible role of magnetic fields. Streams, he believes, would be very viscous. Over limited ranges of the possible conditions in gas streams, the particle-dynamics results may approximately represent the true flow - but this must be thoroughly tested in each case.

Popper: Dr. Huang is anxious to comment on this.

Huang: Dr. Aller mentioned things that were discussed in my paper, so I would like to make a few comments. The version of my paper that was circulated, which Dr. Aller refers to, is my first draft. The final version is quite a bit different. I have discussed the hydrodynamical nature of the problem, but there is the great difficulty of how to impose the boundary conditions as I explain in my review paper (final version). You have to assume a certain physical state in order to derive some approximate solutions, because of this difficulty. You have seen the results of Dr. Kitamura who has produced such a solution by the hydrodynamic approach. If you compare his result with my result derived from the three-body particle problem, you find that they agree very well. This shows that in this case particle dynamics is not a bad approximation at all, because the flow is laminar - that point was not dealt with in my final manuscript. Thus, particle dynamics has the advantage of being very simple, while hydrodynamics is the correct approach to the problem. But, I emphasize that hydrodynamics gives no satisfactory solution at present, because of the difficulty with boundary conditions. 
Biermann: I was asked to comment on this letter, and I would say I agree with almost all of it, except the part on viscosity. It's not important to know the Reynolds' number involved. In my paper (1971) I discussed that point briefly. It's very evident that the Reynolds' number is very large (many powers of 10) and I would say the flow is turbulent. Turbulence is very difficult to treat, and, of course, has to be neglected in nearly all the approximations we can make at the moment. The same reasoning makes the thermal conductivity probably unimportant.

Chen: I agree with Dr. Biermann about the large value of Reynolds' number that one would find for circumstellar flow. Hence, as in the case of flow around Be stars, the viscous terms are many orders of magnitude smaller than the inertial term.

Kitamura: I would like to comment on two points. First, in treating the problem of gaseous rings in close binary systems the use of particle dynamics may not be best. Even so, for practical application it would perhaps be alright as the first approximation, because as I showed from hydrodynamic equations*, the pressure-term of the form $\partial P / \partial \xi$ that appears in the solution is found to vanish at the maximum gas density within the ring. Secondly, in the hydrodynamic approach to the present problem it would be important to find a device by which the mathematical complexity can be reduced as far as possible. What $I$ would like to mention is that the Roche coordinates would be a most convenient means for mathematical treatment in this sense. By using these coordinates, the surface of a parent star with an emission ring in a close binary can be best approximated by one of the zero-velocity surfaces on which the boundary conditions can be set in much more tractable form.

Biermann: I did some unpublished work on two-dimensional subsonic flow, and it turned out that the numerical complexity involved in expressing the boundary conditions nicely is so great that my impression, at the time, was that the advantage of the proper coordinates is offset by the complexity. It was almost impossible to check the computer code.

Popper: It's exactly 5.30 p.m., the time set for the termination of this session. Perhaps it would be a good time to finish.

\section{References}

Batten, A. H.: 1967, Publ. Dominion Astrophys. Obs. 13, 119.

Biermann, P.: 1971, Astron. Astrophys. 10, 205.

Eggen, O. J., Kron, G. E., and Greenstein, J. L.: 1951, Publ. Astron. Soc. Pacific 62, 171.

Gaposchkin, S.: 1951, Astron. J. 51, 109.

Hall, D. S. and Garrison, L. M.: 1969, Publ. Astron. Soc. Pacific 81, 771.

Kraft, R. P. and Demoulin, M.-H.: 1967, Astrophys. J. Letters 150, L183.

Kristian, J., Sandage, A. R., and Westphal, J. A.: 1967, Astrophys. J. Letters 150, L99.

Kuiper, G. P., Struve, O., and Strömagren, B.: 1937, Astrophys. J. 86, 570.

Lucy, L. B.: 1967, Astron. J. 72, 813.

Moore, J. H. and Neubauer, F. J.: 1948, Lick Obs. Bull. 20, 1.

Paczyński, B.: 1965a, Acta Astron. 15, 198.

Paczyński, B.: 1965b, Acta Astron. 15, 89.

* See p. 107. 
Paczyński, B. and Ziołkowski, J.: 1968, Acta Astron. 18, 255.

Walter, K.: 1950, Astron. Nachr. 279, 1.

Walter, K.: 1951, Astron. Nachr. 280, 149.

Walter, K.: 1954, Astron. Nachr. 282, 122.

Wilson, R. E.: 1970, in L. Gratton (ed.), 'Non-Solar X- and Gamma-Ray Astronomy', IAU Symp. 37, D. Reidel, Dordrecht, p. 242.

Wilson, R. E.: 1971, Astrophys. J. 170, 529.

Zahn, J.-P.: 1966, Ann. Astrophys. 29, 313, 489, 565. 